\title{
Development of Complex Energy Saving Device
}

\author{
Kwi-Joo Lee* and Jung-Sun $\mathrm{An}^{*}$ \\ *Department of Naval Architecture and Ocean Engineering, Chosun University, Gwangju, korea
}

KEY WORDS: Energy saving device, Stator, Complex energy saving device

ABSTRACT: A complex energy saving device has been developed for middle class vessels. The propulsive performance of the developed device is described through a model test. The pre-swirl stator, which recovers the rotational energy of the propeller slipstream, is a well-known energy saving device for large vessels. The pre-swirl stator for a large vessel is usually cast as a part of the stern frame and has a high cost. The manufacture of a cast stator for an existing vessel is almost impossible. The complex device that was developed can be fitted on astern frame by welding. The model tests show a 4-6\% efficiency gain for middle class vessels with the developed appendages compared to those with bare hulls.

\section{Introduction}

From the view point of the ship's operational economy, the improvement of ship performance has been an important topic in ship design and research field due to the increase of fuel prices.

The problem has been generally tackled by the routine ways of ship hydrodynamics, that is, rational principal dimensions, optimization of hull form and adoption of improved propulsive system.

Despite of continuous efforts of scientists and engineers in finding the better hull form, the performance improvement by hull form improvement itself has its limitations in many cases.

So, the extensive activities on the development of energy saving devices are still on their progress and utilized in the actual ships (Lee et al., 1992).

On of the most applicable to energy saving in ships will be preswirl stator for which variations are shown in following Fig. 1.

\section{Conventional Pre-swirl Type Energy Saving Devices}

As shown in Fig. 2, this energy saving device pre-swirl

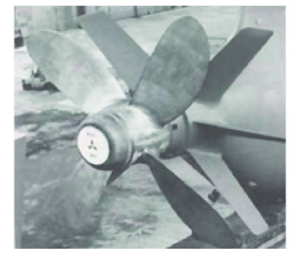

Reaction fin $(\mathrm{MHI})$

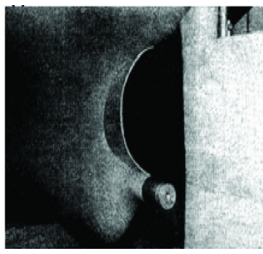

Aperture-Fin (IHIMU)

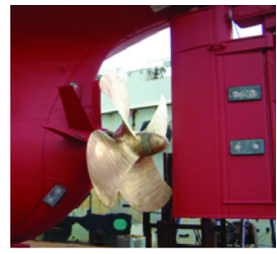

Eco-stator (Fluid Techno)
Fig. 1 Pre-swirl stator
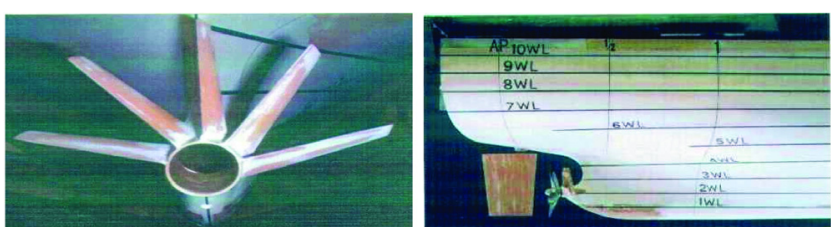

Fig. 2 Conventional pre-swirl type fins
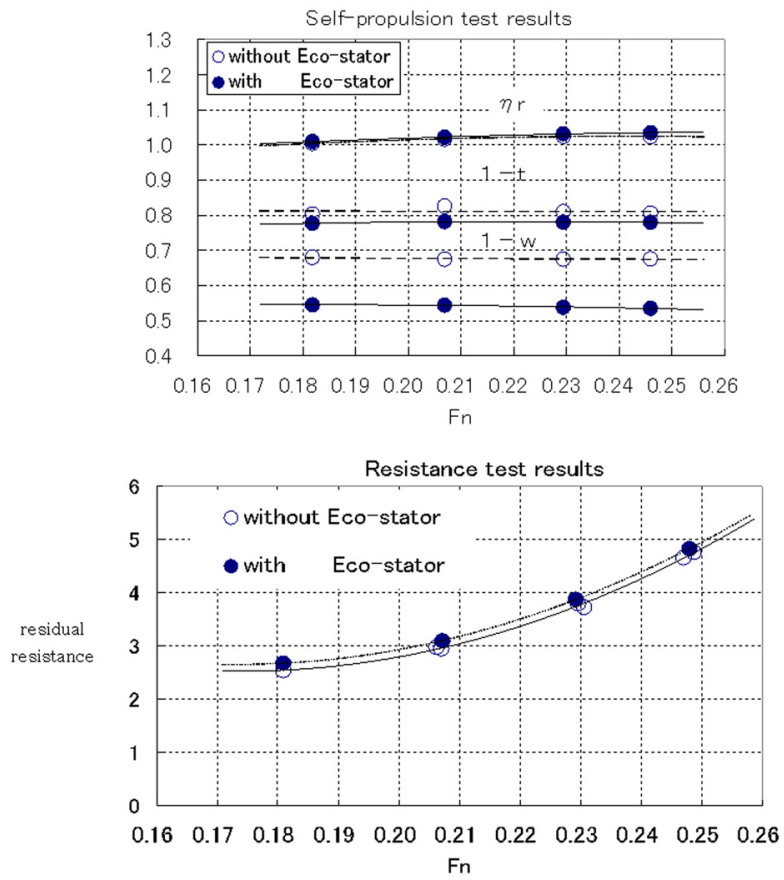

Fig. 3 Comparison of model test results

consists of several fins which have almost the same span length as the propeller radius and are fixed radially on the stern frame in front of the propeller. Fig. 3 represents the example results of the propulsive performance test of a ship,

Corresponding author Jung Sun An: 309, Pilmun-daero, Dong-gu, Gwangju, Korea, +82-62-230-7882, running79@naver.com. 
10,000GT, PCTC (Pure carand truck carrier). It is known that the wake factor

(1-w) decreased considerably. This is caused by the fact that the rotating flow was generated by the fins in front of the propeller plane and the number of propeller revolutions decreased(Asaumi et al., 2006).

\section{Development of a Complex Type Energy Saving Device}

\subsection{Eco-stator}

The maximum span length of the conventional energy saving fin becomes about $1.5 \mathrm{~m}$ from a standpoint of the strength limit. Therefore we could not install it to a ship with the propeller of more than about $4.0 \mathrm{~m}$ in diameter.

Then we improve the conventional pre-swirl type energy saving fin to install for a ship with the propeller of about $5.0 \mathrm{~m}$ in diameter. That is, we cut by half of the span length of the horizontal fins on both sides to control the flow field near the stern frame. The setting angles of the conventional horizontal fins were fixed to control mainly the flow near the fin tip. In this case, the fin did not control properly the flow field near the fin root by the presence of longitudinal vortex due to the ship hull, rather deteriorated the propulsive efficiency. By adopting the horizontal fins of almost half-length of propeller radius, we can control the flow field only near the stern frame and then get almost equal effect to the conventional fin. Moreover, we attach an end plate to the tip of the shorter horizontal fin in order to decrease the tip vortex. Furthermore, we put the bent plate in the hull center plane on the stern frame to generate the counter flow to the propeller turning direction. That is, as shown in Fig. 4, this new pre-swirl type energy saving device consists of four fins on the stern frame and the bent plate in the hull center plane. This device was named "Eco-Stator", which can enhance the safety for a drift obstacle such as wood or timber, and reduce manufacturing cost.

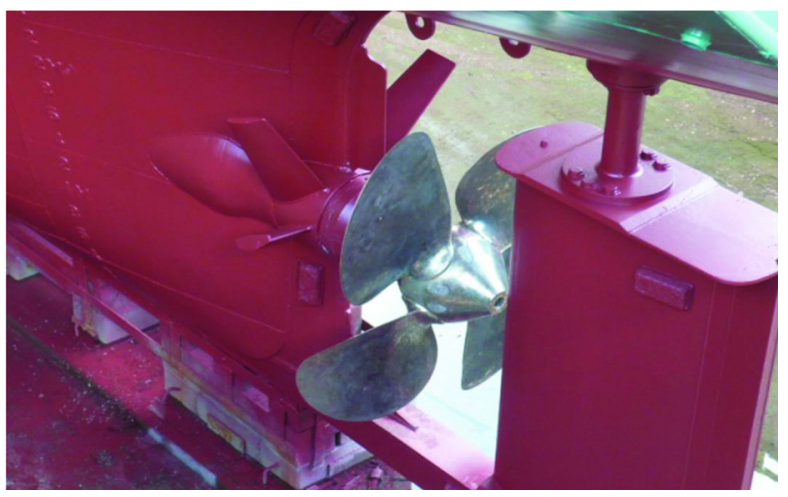

Fig. 4 New pre-swirl stator "Eco-stator"

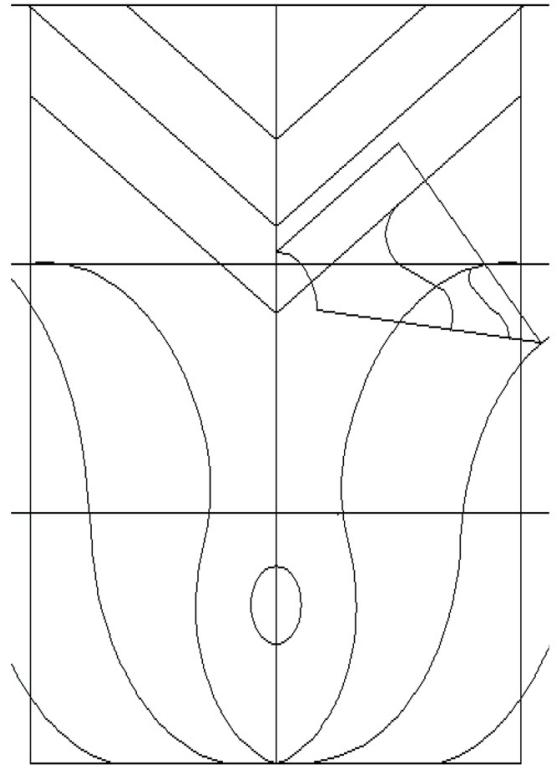

Fig. 5 Asymmetric stern fin

\subsection{Asymmetric stern fin (ASF)}

For the compensation of flow un balancing between port and starboard and for the minimization the span of stator, asymmetric stern fin has been developed as shown in Fig. 5.

\subsubsection{Physical background of the development of asymmetric stern fin}

By the flow visualization technique, for most U-shape stern ships with stern bulbs, we can observe the generation of bilge vortices as a result of three dimensional stern flow separation, thus leading to transverse flow components at the propeller plane. These stern vortices generally result in the inhomogeneities of the flow field at stern and propeller plan. With these inhomogeneities, there are two possible means to improve the propeller intake flow and to recover energy loss by special retrofit devices(Kang et al., 2004 and Kim et al., 1993).

One is to prevent the generation of rotational energy loss in the propeller slipstream by means of flow deflection fins. The other is to locally accelerate the retarded flow by means of tunnel fins above and ducts ahead of the propeller.

When a propeller is rotating clockwise, there occurs dominant flow separation on port side above the propeller shaft and the propeller induces prerotational flow, leading to asymmetric flow behavior. With these physical phenomena in mind, asymmetric stern fin is developed to control vorticities and rotational flow in the vicinity of propeller tip following comparative model tests in the towing tank and flow visualization tests by means of a tuft grid in the circulating water channel.

In the vicinity of propeller, a pair of vortices is generated 

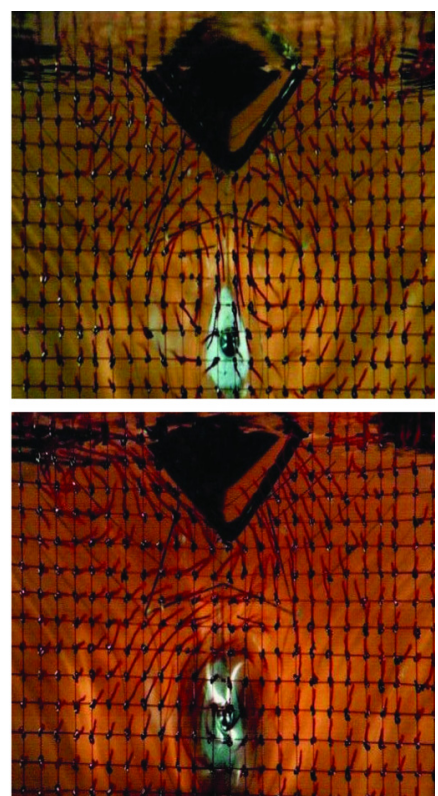
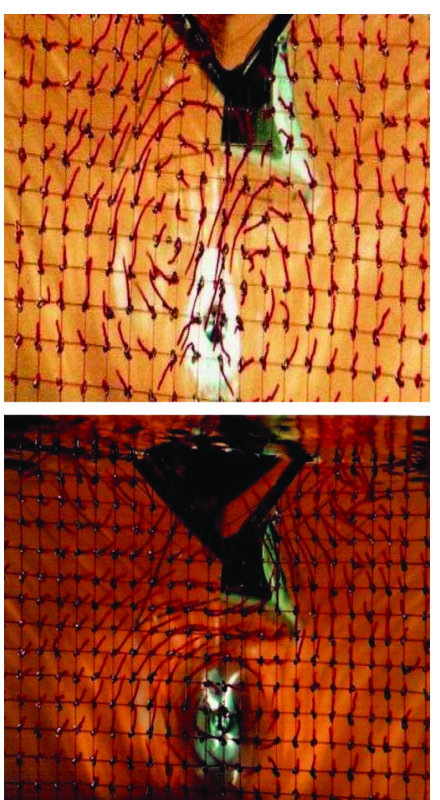

Fig. 6 Wake visual test with tuft grid at propeller plane(without Fig. 7 Wake visual test with tuft grid behind propeller plane propeller) (with propeller)

from the bilge at stern and give rise to viscous pressure resistance, i.e., energy loss. Moreover, the stern flow generated from these vortices moves upward on both ship sides and moves downward near the center plane of the ship, leading to difference angle of attack of propeller blades on port and starboard side which may reduce the propeller efficiency.

Against the stern flow mechanism, the design of the stern fin generally plays a role as flow guiding device making a wake field near the upper part of the propeller plane uniform by both deflecting flow toward propeller and reducing the wake peak at propeller top position.

When the propeller rotates in one specific direction, its performances on port and starboard side are different and the pressure center shifts to the side where the propeller blades move downwards.

This in turn leads to a asymmetric pressure distribution on the hull in front of the propeller, which influences the boundary layers on port and starboard side differently. The idea in the design of asymmetric fin is to reflect the stern vortices which move upwards in the region the propeller rotates downwards. This reflecting may reduce the wake near propeller tip and contribute to the equalization of wake field, thus may lead to a positive gain in propulsion efficiency(Lee et al., 1992).

To clarify the effects of asymmetric fin on propulsion efficiency flow visualization test by means of a tuft grid as shown in Fig. 6 and 7 were carried out, which presents the transverse velocity component in the propeller plane. Fig. 6 and 7 reveals a separation of flow in front of respectively. In case of a clockwise turning more separation occurs on port side above the propeller shaft where the propeller blades move upwards. The flow becomes turbulent and is even partly deflected in the opposite direction. From this point of view developed appendage might contribute towards the reflection of vortex flow which moves upwards in the region where propropulsive efficiency might be occurred.

The full scale wake is calculated from the model wake $W_{T M}$, and the thrust deduction $t$ :

$$
W_{T S}=(t+0.04)+\left(W_{T M}-t-0.04\right) \times \frac{C_{F_{s}}+C_{A}}{C_{F_{M C}}}
$$

In the formula, the factor 0.04 is used to take account for rudder effect. If full scale wake

$W_{T S}$ is greater than model wake $W_{T M}$, following formula is used.

$$
W_{T M}=W_{T S}
$$

The wake diagram at full load condition obtained from towing tank is shown in Fig. 8.

Stator angle of attack and position had been decided based upon the CFD calculation results inflow angle and direction of flow at stator position.

\subsubsection{Results of performance evaluation tests}

Self propulsion tests results are analysis by modified ITTC 78 prediction method.

The results of the propulsion test for ASF in the towing tank are summarized in Table 1 for the speed of 14 Knots. peller blades move downwards, by which the increase of 


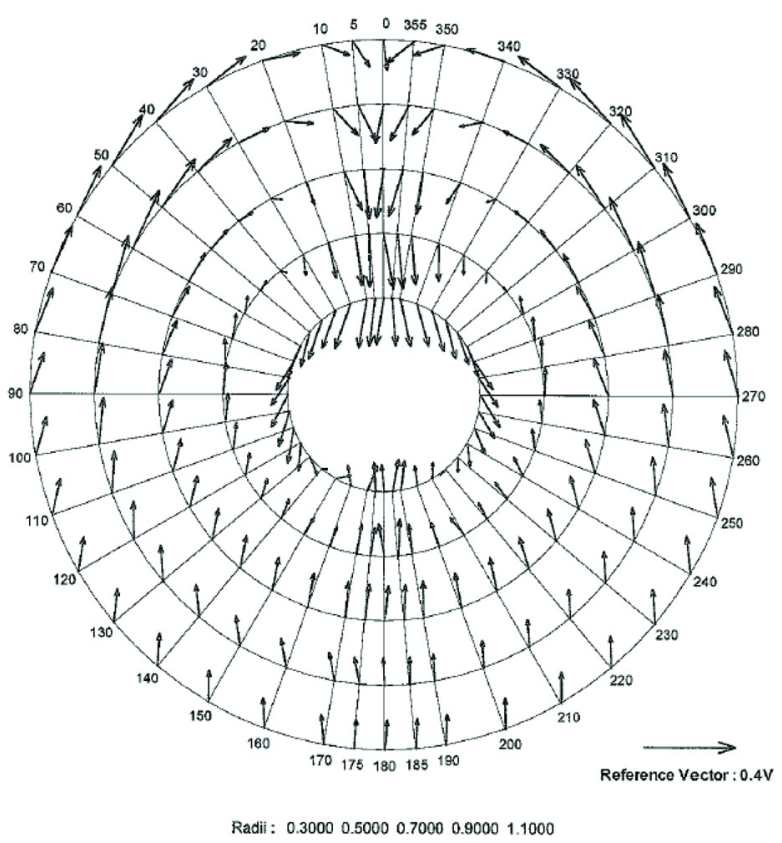

Fig. 8 Wake measurement result at TT(towing tank)

Table 1 Comparison of the results of propulsion test with and without asymmetric stern fin (ASF)

\begin{tabular}{ccc}
\hline \hline & Bare hull & With ASF \\
\hline Thrust deduction fraction & 0.226 & 0.204 \\
Ship wake fraction & 0.297 & 0.301 \\
Hull efficiency & 1.101 & 1.139 \\
Relative rotative efficiency & 1.028 & 1.020 \\
$\eta_{D}$ & 0.656 & 0.673 \\
\hline
\end{tabular}

The results show the reduction of trust deduction fraction $(t)$ and wake fraction $(w)$ for asymmetric stern fin. leading to increase of hull efficiency $\left(\eta_{H}\right)$. The relative rotative efficiency $\left(\eta_{R}\right)$ decrease by 2 percent. It seems that this low $\eta_{R}$ is the effect of relatively worse irregular wake field by the rotation of propeller.

\section{Verification of Energy Saving Effect of "Complex Appendage"}

The complex appendage, combination with eco-stator and ASF is shown in Fig. 9.

By using model ship of 50k tanker, we examine the effect of "Complex Appendage". Table 2 shows comparisons of resistance and self propulsion factors with and without "complex appendage". With "Complex Appendage", we knowthat the wake factor decreases remarkably and the hull

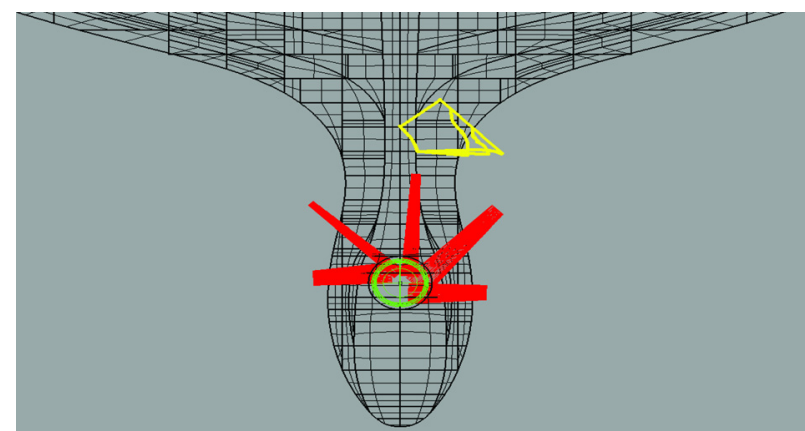

Fig. 9 Configuration of complex appendage

Table 2 Comparison of self propulsion test results with and without "Complex Appendage"

\begin{tabular}{ccc}
\hline & Bare hull & $\begin{array}{c}\text { complex } \\
\text { appendage }\end{array}$ \\
\hline Trust deduction fraction & 0.226 & 0.180 \\
Ship wake fraction & 0.297 & 0.354 \\
Hull efficiency & 1.101 & 1.27 \\
Relative rotative efficiency & 1.028 & 1.010 \\
$\eta_{D}$ & 0.656 & 0.686 \\
\hline
\end{tabular}

efficiency increases.

\section{Conclusions}

The function of stern fin appended at the stern of ship is generally considered to equalize wake field at the propeller plane by both directing flow to propeller and reducing the wake peak at top position. When the propeller rotates in one direction, its performances of port and starboard sides are different and the centerof pressure shifts to the side where the propeller blades downwards. This in turn leads to a non-symmetric pressure distribution on the hull in front of propeller, which influences the boundary layers of port and starboard differently.

The asymmetric fin, which is designed to guide the flow for the equalization of wake field, showed $2.5 \%$ of DHP reduction .

And the result of model test with complex appendage shows about $4.5 \%$ power gain.

\section{Acknowledgements}

This study was supported by research funds from Chosun university 2011. 


\section{References}

Asaumi. T., Ikeda. T., Tamashima. M. and Shinohara. H. (2006). "Effectiveness of an Energy Saving Device 'Friend Fin', Conference Proceedings". The Japan Society of Naval Architecture and Ocean Engineering, Vol 3, pp 261-264.

Kang, Y.D., Kim, M.C., and Chun, H.H. (2004). "A Study on the Design of Biased Asymmetric Pre-swirl Stator Propulsion System", J. of Society of Naval Architects of Korea, Vol 41, No 3, pp 13-21.

Kim, M.C., Lee, J.T., Suh, J.C. and Kim, H.C. (1993). “A Study on the Asymmetric Preswirl Stator System" J. of Society of Naval Architects of Korea, Vol 30, No 1, pp 30-44.
Lee, K.J., Joa, S.W., Parkm M. and Lee, Y.W. (1992). “An Experimental Study on the Ship Performance Improving Appendages" , Hull Form 92 Proceeding , 1.60-1.67.

Lee, J.T., Kim, M.C., Suh, J.C., Kim, S.H. and Choi, J.K. (1992). “Development of a Preswirl Stator-Propeller System for Improvement of Propulsion Efficiency : a Symmetric Stator Propulsion System", Transactions of the Society of Naval Architects of Korea, Vol 29, No 4, pp 132-145.

2011년 5월 19일 원고 접수

2011년 9월 5일 심사 완료

2012년 6월 21일 게재 확정 